with their level of disturbance. The hard to place group scored very highly, while the own home group scored lowly. In between these two were those assigned to the 24-hour staffed hostel and to group homes. The former were more disturbed (on the top four scale) than the latter.

The objection that can be raised is that this association could be spurious, because the ward team decided placement and rated the behaviour. However, it should be remembered that the placements were determined some months before the behaviours were rated. The other related factor is that the placements were discussed by a consultant psychiatrist, junior doctor, social worker and nurses. The behaviour rating was performed by nurses alone. Further, because of the time lag, the nursing staff had changed to some extent. Moreover, they performed these ratings 'blind' to the placement decisions made earlier.

\section{Conclusion}

The subculture of community carers differs from that of ward nurses. Sometimes the ideology of the former is such that they do not wish to identify problem behaviour. There is need of a simple medium whereby one group can communicate with the other about patients who will become residents. This very simple survey tool, estimating the level of behavioural disturbance, provides this means of communication.

\section{Acknowledgements}

Thanks are due to Dr R. Perkins and Mr A. Copello (Department of Psychology, Springfield Hospital, London) for their provision of the survey tool; to Dr R. L. Symonds and to Professor T. K. J. Craig for their helpful suggestions on the manuscript; to $\mathrm{Dr} \mathrm{H}$. Gibson and $\mathrm{Mr} \mathrm{H}$. Green for their assistance with word processing; to the nurses and doctors of Bexley Hospital for help with the survey, especially Drs E. Parker and S. Cope.

\section{References}

BAKER, B. \& HALL, J. N. (1983) REHAB - Rehabilitation Evaluation Hall and Baker. Aberdeen: Vine.

Clifford, P., Charman, A., Webb, Y. \& Best, S. (1991) Planning for community care. Long-stay populations of hospitals scheduled for rundown or closure. British Journal of Psychiatry, 158, 190-196.

HoNigfeld, G. \& KLETT, C. J. (1965) The nurses' observation scale for input evaluation. Journal of Clinical Psychology, 12, 65-71.

WHO (1988) WHO/DAS, Psychiatric Disability Assessment Scale. Geneva: World Health Organization.

WING, J. K. \& Brown, G. W. (1970) Institutionalism and Schizophrenia. Cambridge University Press.

\title{
Hostages returned from the Gulf
}

Recent reports show that continuing problems are suffering continuing stress as a result of traumatic being experienced by the returned hostages and experience.

their families. The Department of Health advised There is a further resource available within the at the time of the hostages return that, apart from system in the form of a list of psychiatrists with those who needed immediate emergency treatment, special expertise and experience in this field. The the initial point of referral ought to be the persons list was circulated to Regional Medical Officers in general practitioner. General practitioners in many January, 1991 and those listed are available to offer cases now employ their own staff who are able to tertiary level opinions and guidance if necessary. Any offer support and counselling. In some cases the psychiatrist who wishes to have a copy of this list general practitioner may, however, decide that the should contact the Secretary, Mrs V. Cameron.

most appropriate response is referral to the psy-

chiatric service. All local psychiatric services

should be able to respond to the needs of people September 1991

Professor ANN Gath

Registrar 